\title{
Deep Belief Network for Prediction of Rician Fading Channel
}

\author{
Venkatesh P, Saikat Majumder
}

\begin{abstract}
In this paper a novel channel prediction scheme is presented for rician fading channel. The channel prediction is done by using a Deep Belief Network (DBN) which is composed of two Restricted Boltzmann Machines (RBMs), this deep learning algorithm can produce fewer predictive errors than echo state networks and other predictive approaches.. Simulation results shows that the DBN channel prediction system has a lower NMSE than the prediction of the echo state network and other conventional prediction methods and the obtained SER gap between the actual CSI and predicted CSI is small.
\end{abstract}

Keywords: Channel prediction, Deep Belief network, Restricted Boltzmann Machine, Rician fading.

\section{INTRODUCTION}

In future wireless communication $(5 \mathrm{G})$ the demands like high speed transmission, high reliability, quick access and strong security communication are put forward and also in the wireless communication systems the channel state changes dynamically. Hence to meet the requirements like high efficiency and high speed transmissions the adoption of new technologies should be considered. A major limitation for the wireless communication systems is Channel State Information (CSI) estimation and the estimation of this CSI by frequency division duplexing is outdated so the CSI knowledge at the transmitter is predicted by channel prediction techniques. The information of the channel will be changing continuously due to the location change of the user's receiver, which is the difficulty for the base station to achieve beam-forming etc, so the key point to such problems is the channel prediction technique.

There is several channel prediction strategies have been employed till now, certainly one of them is the linear channel prediction model. The AR model in [1] which is autoregressive model is turned into followed to explain the channel impulse response, where in a linear combination of current CSIs and past CSIs was expressed. There may be one greater channel prediction class which is a nonlinear channel prediction. By means of the usage of a complex-valued neural network an accurate time-various channel prediction technique is presented in [2]. It indicates to a decrease bit errors rate (BER) than different conventional strategies. The direction loss forecasting in plane cabin scenarios is employed by using the support vector device (SVM) in [3]. To predict the strength of the obtained signal in the fast

Revised Manuscript Received on December 12, 2019

* Correspondence Author

Venkatesh $\mathbf{P} *$, Department of Electronics and Communication Engineering, National Institute of Technology, Raipur, India. venkatesh0696@gmail.com

SaikatMajumder, Department of Electronics and Communication Engineering, National Institute of Technology, Raipur , India. smajumder.etc@nitrr.ac.in various surroundings the multi-decision wavelet is introduced in [4]. In aggregate with the AR and linear regression (LR) version the discrete wavelet transform (DWT) technique is used to predict all of the coefficients. The DWT-AR-LR [4] set of rules outperforms the conventional linear AR technique..

A DBN is the Deep Belief Network and it a class of deep neural network consists of many hidden units, which are connected between the layers but not between the units within each layer, it is the first effective deep learning algorithm in which it is trained greedily in one layer at a time [13]. Thus DBN is used as prediction model and It has deep hidden layers which is an important advantage and is suitable for obtaining lower NMSE and the channel prediction. In this literature, it is shown that DBN is used as solving the channel prediction [6], time series prediction [7], non-linear system modeling [8], satellite channel equalization [9] and spectrum prediction [10] etc. Till now there is no other channel prediction work has been reported on DBN. A novel DBN model is anticipated to report the working of prediction of the channel, which is the most important challenge thinking about the situation of standard theory of implementation. Similarly, to obtain a great performance the specification of components of DBN must be designed and optimized, that are also considered inside the design. On this paper, we suggest a unique channel prediction technique.

In this paper, we propose a novel channel prediction approach for Rician fading channel by using the DBN model. The two layers of RBMs are stacked up on one above another and the DBN is constructed. The classical particle swarm optimization algorithm (PSO) is used to define the DBN structure by optimizing the number of neurons in the RBMs ' visible and hidden layer units and the learning rates are defined so that the pre-training of the DBN should be fast and effective, here the learning is done in the greedy layer wise unsupervised way, so that the units will get updated by using the probability function and biases of the neurons are also obtained and the learning of the weights is done by the Contrastive Divergence method so that the proper weights are obtained by updating the weights of the RBM using the this CD algorithm. After the completion of the Pre-training the fine tune is done by the back propagation (BP) algorithm so that the fast channel prediction of the Rician fading channel is predicted. Finally the NMSE is evaluated for various prediction methods like the echo-state network and the DBN, and the Symbol Error Rate is compared with the perfect and the predicted CSI for the quadrature phase shift keying (QPSK) by simulation 


\section{CHANNEL MODEL}

The Rician slow fading channel is studied on this section. Both the multiple scatter paths and the Line of Sight (LOS) exists between the transmitter and the receiver. We have taken into consideration that both the transmitter and the receiver are constructed with one antenna. In [10] the rician fading channel is modelled between the transmitter and the receiver as

$h(t)=R e^{j\left(2 \pi f_{d} t+\varphi\right)}$

where $\mathrm{R}$ refers the magnitude of Rician fading, and in slow fading it is represented as random variable and the PDF (probability density function) given by,

$p(r)=\frac{2(K+1)}{\alpha} e^{-K-\frac{(K+1) r^{2}}{\alpha}} I_{0}\left(2 r \sqrt{\frac{K(K+1)}{\alpha}}\right)$

Where in $\mathrm{r} \geq 0$ and the $K=\frac{p^{2}}{2 \sigma^{2}}$ suggests the Ricean factor, and $\alpha=P^{2}+2 \sigma^{2}$ represents the overall or total power of line of sight and other scattered components. Here the mean powers of the LOS component and the scattering component $P^{2}$ and $2 \sigma^{2}$ and $I_{0}(\cdot)$ refers the primary type of zero order modified Bessel function.

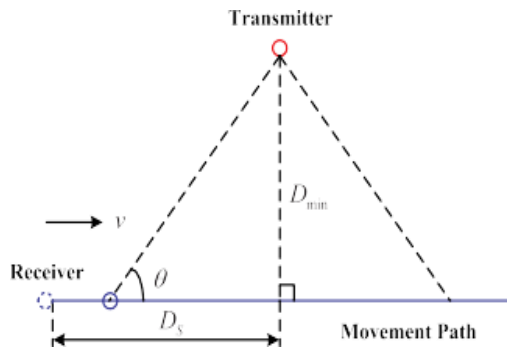

Fig. 1. Geometrical figure for calculating $\cos \theta$

Furthermore, the parameter $\varphi$ in (1) denotes the LOS component's phase offset, it is a random variable and $\mathrm{fd}$ is the Doppler shift of LOS component. The $\mathrm{fd}$ is expressed as,

$$
f_{d}=\frac{v}{c} f_{c} \cos \theta
$$

Where the speed or velocity of the consumer is expressed in $\mathrm{v}, \mathrm{c}$ indicates the velocity of an EM wave and fc is the frequency of the carrier. The component located in $\theta$ (3) is the LOS component's angle of arrival. As illustrated in Fig. 1, $\cos \theta$ value can be obtained as,

$\cos \theta=\left\{\begin{array}{l}\frac{D_{s}-v t}{\sqrt{D_{\text {min }}^{2}+\left(D_{s}-v t\right)^{2}},} \quad 0 \leq v t \leq D_{s} \\ -\frac{v t-D_{s}}{\sqrt{D_{\text {min }}^{2}+\left(v t-D_{s}\right)^{2}}}, \quad v t>D_{s}\end{array}\right.$

Where Ds refer the preliminary distance between sender and receiver in the transferring route of the person and Dmin is gap among the transmitter and motion course. without generality loss, the motion course is to be the immediate line.

We assume that $\mathrm{h}(\mathrm{t})$ is sampled in equation (1) and $\mathrm{t}=\mathrm{kTs}$, $\mathrm{k}=1,2, \ldots$ Where Ts refers to sampling time. The sample of $\mathrm{h}(\mathrm{t})$ is therefore defined as

$h\left(k T_{s}\right)=R e^{j\left(2 \pi f_{d} k T_{S}+\varphi\right)}$

\section{CHANNEL PREDICTION USING A DBN}

\section{A. $\boldsymbol{R B M}$}

RBM is a bipartite graphical model and it is the building block of the DBN. More technically an RBM is a stochastic neural network (stochastic means the activations has a probabilistic element) and it is an energy based network which is composed of couple of layers, that is visible and hidden layer, in which it is trained by unsupervised learning. We restrict the network so that the visible units are not connected to each other and the hidden units are not connected to each other to make learning easier. The below Fig. 2 shows the typical RBM model.

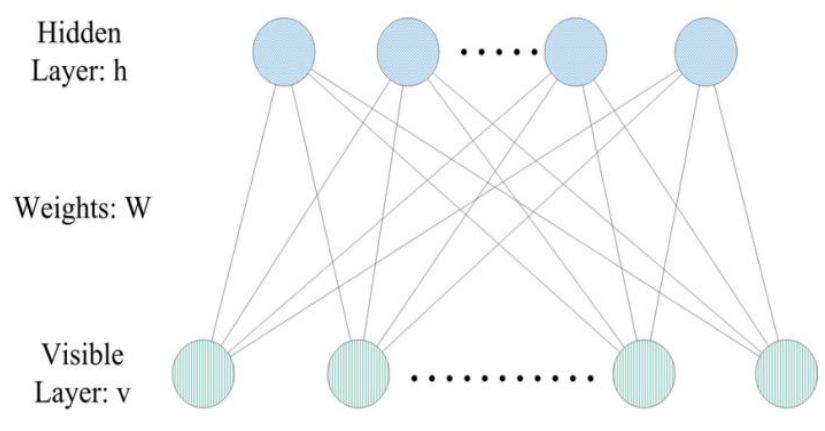

Fig. 2. Structure of RBM

The advantage of RBM is that it is the reconstruction oriented learning. Here the features are extracted through the learning of information in hidden units and the input is reconstructed from the hidden units. The actual input is reconstructed or recovered from the reconstruction process, shows that the input information is reserved in the hidden units and the weights and biases are updated so that it is capable to measure the input data.

\section{B. Dbn Model}

DBN is the Deep Belief Network which is the probabilistic generative model in which the RBMs are stacked upon one above another as shown in Fig. 2, Which is used to design the structure effectively as a representation of the function (data input) [12]. A logistic layer above DBN for supervised learning is applied to solve problems such as classification and regression. DBN training is therefore performed in two phases, namely pre-training and fine tuning.

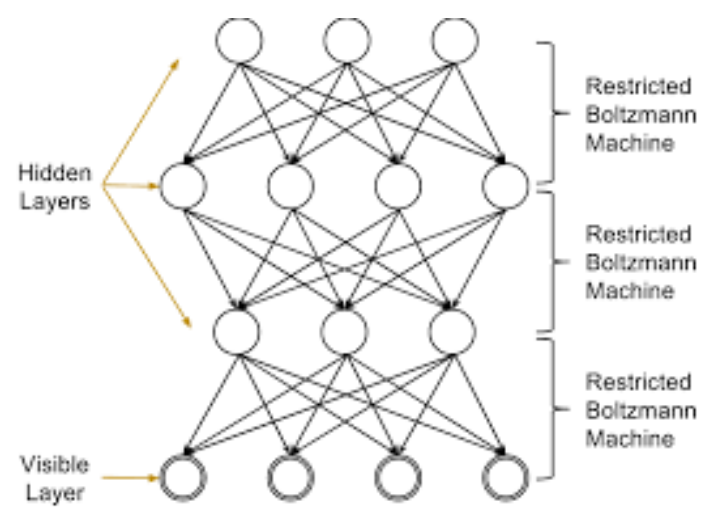

Fig. 3. Structure of DBN with stacks of RBM 
Deep Belief Network is pre-trained in a greedy, unsupervised way in the first step, beginning with the first layer of RBM and using its outputs as the inputs to train the next RBM after training current RBM. The outputs of the last $\mathrm{RBM}$ are the features learned during the pre-training process once all RBMs have been trained. It is agreed that for deep learning, quick and efficient pre-training is extremely important and beneficial.

The BP algorithm is used in second phase to fine-tune network and to combine the neural network layers using the learned features. It could find a minimum of parameters initialized through DBN in a peripheral region. Finally, you can use the trained DBN to predict the channel.

\section{Channel Prediction Scheme}

The DBN learning is done as a greedy layer wise method that's much like the pre-training which is cited above. However, once the training is carried out, DBN is itself fixed and constant. Then, the description of the DBN learning is as follows.

Since each RBM only has the best visible and hidden binary units, it is considered to be a special form of random Markov filed with visible units $\mathrm{v}=\{0,1\} \mathrm{D}$ and hidden units $\mathrm{h}=\{0,1\} \mathrm{F}$. The energy of the unit's joint configuration is calculated by RBM's weights and biases and is given by

$$
\begin{aligned}
& E(v, h ; \theta)=-\sum_{i=1}^{D} a_{i} v_{i}-\sum_{j=1}^{F} b_{j} h_{j} \sum_{i=1}^{D} \sum_{j=1}^{F} w_{i j} v_{i} h_{j} \\
=- & a^{T}-b^{T} h-v^{T} W h
\end{aligned}
$$

Where in the model parameters $\theta=\left\{a_{i}, b_{j}, w_{i j}\right\}, w_{i j}$ refers to the weight between the visible unit $\mathrm{i}$ and the hidden unit $\mathrm{j}$; $a_{i}$ and $b_{j}$ are visible and hidden unit bias parameters respectively. The probability of a visible-hidden vector pair $(\mathrm{v}, \mathrm{h})$ is linked to the function of energy and is defined as

$p(v, h ; \theta)=\frac{1}{Z(\theta)} \exp (-E(v, h ; \theta))$

Where the $Z(\theta)$ is the normalizing component, that is given by the below eqn (8).

$$
Z(\theta)=\sum_{v} \sum_{h} \exp (-E(v, h ; \theta))
$$

By adjusting $\theta$ the probability of the training vector is improved and the probability to all input vectors is given by the RBM thru energy function as shown in eqn(6)

The logistic function gives the conditional probabilities corresponding to the values of $i$ and $j$ which are visible and hidden unit respectively

$\mathrm{p}\left(\mathrm{h}_{\mathrm{j}}=1 \mid \mathrm{v}\right)=\sigma\left(\mathrm{b}_{\mathrm{j}}+\sum_{\mathrm{i}} \mathrm{v}_{\mathrm{i}} \mathrm{w}_{\mathrm{ij}}\right)$

$\mathrm{p}\left(\mathrm{v}_{\mathrm{i}}=1 \mid \mathrm{h}\right)=\sigma\left(\mathrm{a}_{\mathrm{i}}+\sum_{\mathrm{j}} \mathrm{h}_{\mathrm{j}} \mathrm{w}_{\mathrm{ij}}\right)$

Where $\sigma(\cdot)$ is the logistic sigmoid function, that is, $\sigma(\mathrm{x})=$ $1 /(1+\exp (-x))$. The input information can be reconstructed by setting the $v_{i}$ to 1 with the possibility of the state of the hidden units is given eqn(10). Then the states of the secret units are up-to-date to reflect the characteristics of the reconstruction.

A method of contrastive divergence (CD) is used to know w. The weight update rule is given by
$\Delta w_{i j}=\eta\left(\left\langle\mathrm{v}_{\mathrm{i}} \mathrm{h}_{\mathrm{j}}\right\rangle_{\text {data }}-\left\langle\mathrm{v}_{\mathrm{i}} \mathrm{h}_{\mathrm{j}}\right\rangle_{\text {recon }}\right)$

where $\eta$ is the learning rate. By learning, we can acquire the correct value of $\mathrm{w}$. The process is repeated till the weights of all RBMs are updates, so that we are able to gain a trained DBN.

\section{SIMULATION RESULTS AND ANALYSIS}

The simulation results of the channel prediction proposed are evaluated in this section and the output is analyzed. The partial values of the parameter are considered in [16],[17] as $\mathrm{P}=\sqrt{6}, \sigma=1, \mathrm{v}=5 \mathrm{~m} / \mathrm{s}, \mathrm{c}=3 \times 10^{\wedge} 8 \mathrm{~m} / \mathrm{s}, \mathrm{fc}=2 \mathrm{GHz}, \mathrm{DS}=$ $500 \mathrm{~m}, \mathrm{Dmin}=50 \mathrm{~m}, \mathrm{M}=5, \mathrm{Ts}=1 \times 10^{\wedge}-2 \mathrm{~s}$, Ns $=500$, and $\mathrm{Nc}=21$. Meanwhile, by jointly considering the prediction error metrics in [5] and the NMSE is defined as,

$N M S E=\frac{\sum_{n=N_{t}+1}^{N_{S}} \mid h\left((n+M) T_{s}-\widehat{h}\left(\left.(n+M) T_{S}\right|^{2}\right.\right.}{\sum_{n=N_{t}+1}^{N_{S}} \mid h\left(\left.(n+M) T_{S}\right|^{2}\right.}$

Where $\hat{h}\left((n+M) T_{s}\right.$ is the predicted CSI, and where it is the DBN output. The perfect CSI in the test samples is $h((n+$ MTs

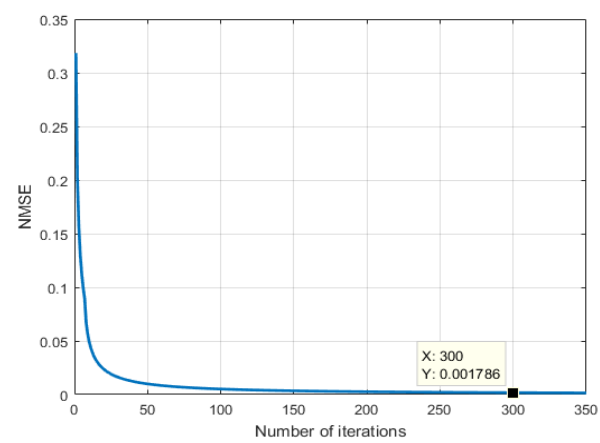

Fig. 4a. NMSE versus Number of iterations under 20-20-1 neurons.

In the above figure $4 \mathrm{a}$ the plot between the NMSE and the number of iterations is done under the 20-20-1 neurons, i.e there are 20 input units, 20 hidden units and 1 output units in the network which shows that NMSE is $9.5124 \mathrm{e}-4$ that is very much lesser than the echo state network. The simulation is performed in MATLAB software.

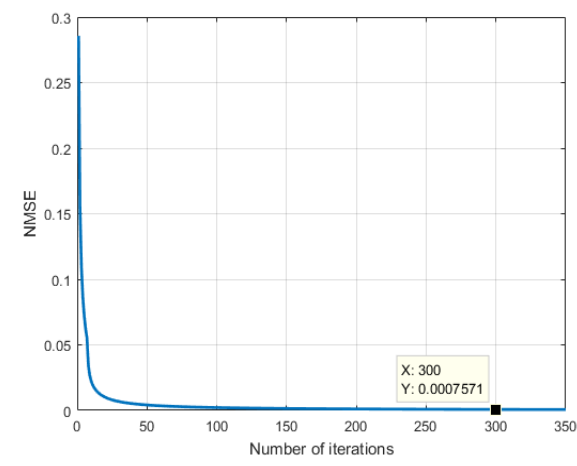

Fig. 4b. NMSE versus Number of iterations under 40-40-1 neurons. 
In the above figure $4 \mathrm{~b}$ the plot between the NMSE and the number of iterations is done under the 40-40-1 neurons, i.e there are 40 input units, 40 hidden units and 1 output units in the network which shows that NMSE is $7.134 \mathrm{e}-4$ that is very much lesser than the echo state network and the above DBN with 20-20-1.

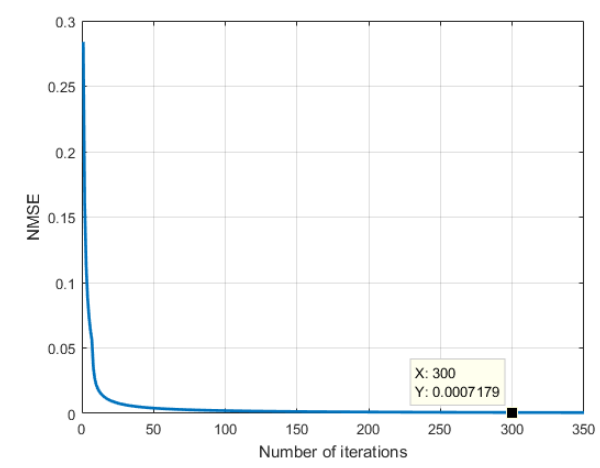

Fig. 4c. NMSE versus Number of iterations under 60-60-1 neurons.

In the above figure $4 \mathrm{c}$ the plot between the NMSE and the number of iterations is done under the 60-60-1 neurons ,i.e there are 60 input units, 60 hidden units and 1 output units in the network which shows that NMSE is $5.174 \mathrm{e}-4$ that is very much lesser than the echo state network and the above DBN with 40-40-1.

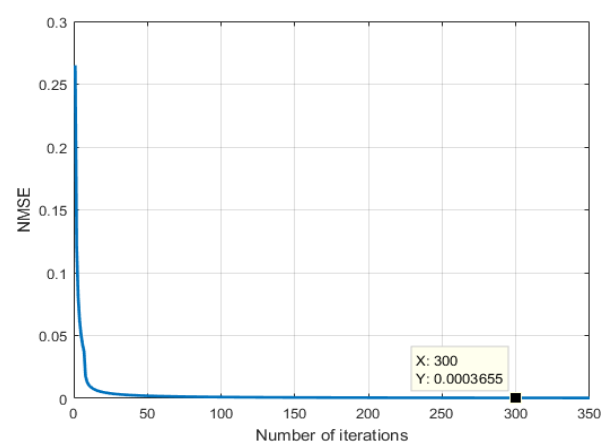

Fig. 4d.NMSE versus Number of iterations under 100-100-1 neurons.

In the above figure $4 \mathrm{~d}$ the plot between the NMSE and the number of iterations is done under the 100-100-1 neurons ,i.e there are 100 input units, 100 hidden units and 1 output units in the network which shows that NMSE is $3.655 \mathrm{e}-4$ that is very much lesser than the echo state network and the above DBN with 60-60-1. But here we are not using this design because this design takes more time consumption and more hardware components is also required.

NMSE is the performance measure of the channel prediction scheme in which the comparison between the DBN and the ESN is shown in the Table 1. Here the NMSE, learning rates, number of iterations or the training samples and the number of neurons is compared the results are showing that the DBM has the lesser NMSE than the echo state network and has the lesser iterations taken obtain the prediction of the channel. The number of neurons in the DBN is calculated by the PSO particle swarm optimization so that the network's structure has the (20-20-1) number of neurons which means 20 visible units and 20 hidden units of first RBM and 20 visible units and 1 hidden unit or the output unit of the second RBM. The Figure 4 shows the NMSE versus number of iterations under various neurons in the DBN so that the NMSE decreases as the number of iterations increases and goes to be stable. When the number of neurons is (20-20-1), the DBN performance of NMSE obtained is satisfactory with the number of iterations as 300 and also when the number of units are increased the NMSE is further reduced.

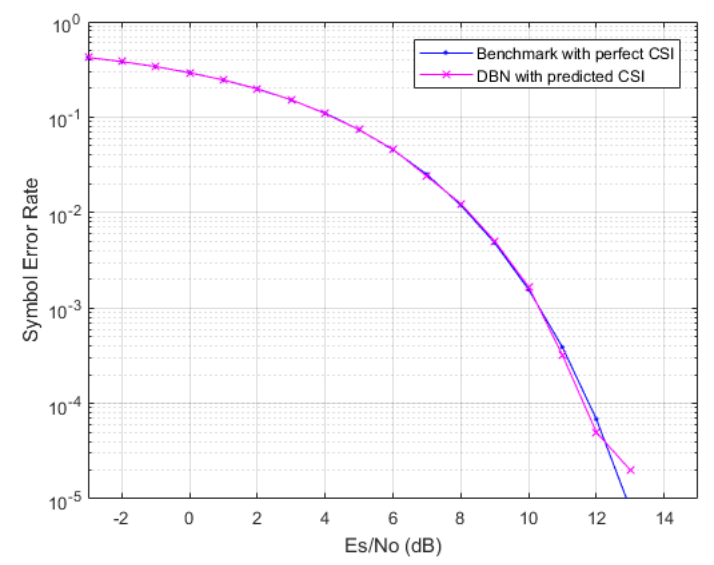

Fig. 5. Assessment of SER in perfect and predicted CSIs

In Fig. 5 the Symbol Error Rate is in comparison with the proper and the predicted CSI for the QPSK via simulation. For signal detection the coherent receiver is adopted. It is via able to be noted that SER is decreased as the $E_{\mathrm{s}} / N_{0}$, which is the spectral density ratio of energy per symbol to noise power, will increase. Furthermore, due to high prediction precision, the SER range between the ideal benchmark CSI and DBN with projected CSI could be very small.

\section{Table 1: Comparison of the DBN over ESN prediction techniques}

\begin{tabular}{|l|l|l|l|l|}
\hline $\begin{array}{c}\text { Structure } \\
\text { and } \\
\text { evaluatio } \\
\text { n model }\end{array}$ & $\begin{array}{l}\text { Echo } \\
\text { state } \\
\text { networ } \\
\mathbf{k}\end{array}$ & \multicolumn{4}{|l|}{ Deep Belief Network } \\
\hline $\begin{array}{l}\text { Number } \\
\text { of } \\
\text { units } \\
\text { (input } \\
\text {-hidden } \\
\text {-output) }\end{array}$ & 50 & $(20-20-1)$ & $(40-40-1)$ & $(60-60-1)$ \\
\hline $\begin{array}{l}\text { Learning } \\
\text { rates }\end{array}$ & 0.3 & 0.47 & 0.47 & 0.47 \\
\hline $\begin{array}{l}\text { Iterations } \\
\text { Nution }\end{array}$ & 2000 & 300 & 350 & 450 \\
\hline NMSE & $4.4 \mathrm{e}-3$ & $9.5124 \mathrm{e}-4$ & $7.134 \mathrm{e}-4$ & $5.174 \mathrm{e}-4$ \\
\hline
\end{tabular}

In addition, it is proved that the ESN performs better prediction than AR,SVM and DWT-AR-LR. This is because ESN has short-term memory capacity, which is an advantage over previous designs and thus producing an accurate prediction. But when ESN is compared with DBN, the DBN has more accurate channel prediction results by using this deep learning technique the feature of feature extraction is done so that the prediction results are more accurate with lesser iterations and pre training of this network is faster and 
effective as well and Because of its high prediction precision, DBN with predicted CSI is very low.

\section{CONCLUSION}

The model proposed is a Deep Belief Network that consists of stacking two Restricted Boltzmann Machines (RBMs) and using classical particle swarm optimization (PSO) to optimize the network structure. Simulation results shows the DBN based channel prediction method has lesser NMSE than the previous designs such as echo state network and other designs, The difference between the expected and actual channel state information of the symbol error rate is very small.

\section{REFERENCES}

1. P. Sharma and K. Chandra, "Prediction of state transitions in Rayleigh fading channels," IEEE Trans. Veh. Technol., vol. 56, no. 2, pp. 416-425, Mar. 2007.

2. T. Ding and A. Hirose, "Fading channel prediction based on combination of complex-valued neural networks and chirp Z-transform,” IEEE Trans. Neural Netw. Learn. Syst., vol. 25, no. 9, pp. 1686-1695, Sep. 2014.

3. X. Zhao, C. Hou, and Q. Wang, "A new SVM-based modeling method of cabin path loss prediction,” Int. J. Antennas Propag., vol. 2013, pp. 1-7, Apr. 2013.

4. X. Long and B. Sikdar, "A wavelet based long range signal strength prediction in wireless networks," in Proc. IEEE Int. Conf. Commun., May 2008, pp. 2043-2047

5. H. Jaeger and H. Haas, "Harnessing nonlinearity: Predicting chaotic systems and saving energy in wireless telecommunication," Sciences, vol. 304, pp. 78-80, Apr. 2004

6. L. Yang, X. Liang, T. Ma, and K. Liu, "Spectrum prediction based on echo state network and its improved form," in Proc. Int. Conf. Intell. Human-Mach. Syst. Cybernet., Aug. 2013, pp. 172-176.

7. ] J. B. Butcher, D. Verstraeten, B. Schrauwen, C. R. Day, and P. W. Haycock, "Reservoir computing and extreme learning machines for nonlinear time-series data analysis," Neural Netw., vol. 38, pp. 76-89, Feb. 2013.

8. B. Zhang, D. J. Miller, and Y. Wang, "Nonlinear system modeling with random matrices: Echo state networks revisited," IEEE Trans. Neural Netw. Learn. Syst., vol. 23, no. 1, pp. 175-182, Jan. 2012.

9. M. Bauduin, A. Smerieri, S. Massar, and F. Horlin, "Equalization of the non-linear satellite communication channel with an echo state network," in Proc. IEEE Veh. Technol. Conf. (VTC Spring), May 2015, pp. 1-5.

10. Y. Zhao, H. Gao, N. C. Beaulieu, Z. Chen and H. Ji, "Echo State Network for Fast Channel Prediction in Ricean Fading Scenarios," in IEEE Communications Letters, volume 21, no. 3, pp. 672-675, March 2017.

11. Y. Chen and N. C. Beaulieu, "Estimation of Ricean K parameter and local average SNR from noisy correlated channel samples," IEEE Trans. Wireless Commun., vol. 6, no. 2, pp. 640-648, Feb. 2007

12. XiaochuanSun, TaoLi, QunLi, YueHuang, YingqiLi, "Deep belief echo-state network and its application to time series prediction," in Knowledge Based Systems, volume 130, pp. 17-29, August 2017.

13. T.Kuremoto, S.Kimura, K.Kobayashi, M. Obayashi, "Time series forecasting using a deep belief network with restricted Boltzmann machines," in Neurocomputing, volume 137, pp. 47-56, January 2014.

14. A. Rodan and P. Tino, "Minimum Complexity Echo State Network," in IEEE Transactions on Neural Networks, volume 22, no. 1, pp. 131-144, January 2011.

15. T. Ding, A. Hirose, "Fading channel prediction based on combination of complex-valued neural networks and chirp Z-transform", in IEEE Transaction on Neural Network Learning Systems, volume 25, no. 9, pp. 1686-1695, September 2014.

16. Y. Chen, N. C. Beaulieu, "Estimation of Ricean K parameter and local average SNR from noisy correlated channel samples", in IEEE Transaction on Wireless Communication., volume 6, no. 2, pp. 640-648, February 2007

17. G. Lu, Q. Zhang, X. Zhang, F. Shen and F. Qin, "CNN BASED RICIAN K FACTOR ESTIMATION FOR NON-STATIONARY INDUSTRIAL FADING CHANNEL," 2018 IEEE Global Conference on Signal and Information Processing (GlobalSIP), Anaheim, CA, USA, 2018, pp. 594-598.

\section{AUTHORS PROFILE}

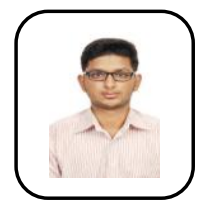

Venkatesh $\mathbf{P}$ received B.E degree in Electronics and Communication Engineering from Sri Sairam Institute of Technology(affiliated to Anna University), Chennai, India in 2018. He is currently pursuing his M.Tech degree in VLSI Design and Embedded Systems at National Institute of Technology, Raipur, India. His current research interest includes Deep Belief Network and its VLSI implementation.

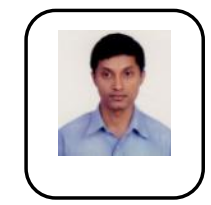

S. Majumderis an assistant professor in the Department of Electronics and Communication at National Institute of Technology, Raipur for last ten years. His research interest includes wireless communication, signal processing and software defined radio. He completed his $\mathrm{Ph}$.D on error correction codes and wireless communication from NIT Raipur and M.Tech degree from NIT Calicut. 